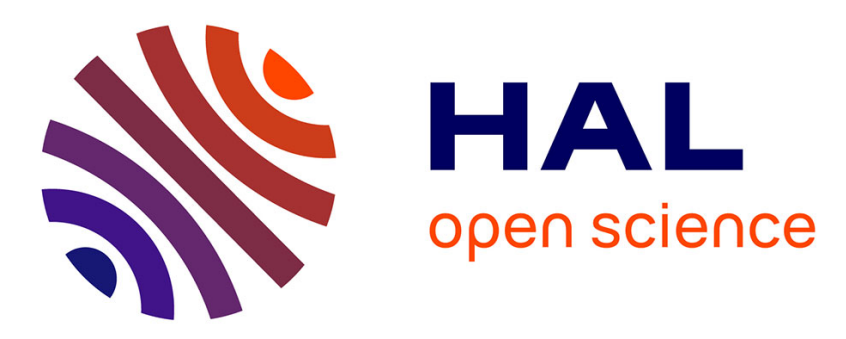

\title{
Profound Degree: A Conservative Heuristic to Repair Dynamic CSPs
}

Yosra Acodad, Amine Benamrane, Imade Benelallam, El Houssine Bouyakhf

\section{To cite this version:}

Yosra Acodad, Amine Benamrane, Imade Benelallam, El Houssine Bouyakhf. Profound Degree: A Conservative Heuristic to Repair Dynamic CSPs. 10th IFIP International Conference on Artificial Intelligence Applications and Innovations (AIAI), Sep 2014, Rhodes, Greece. pp.140-149, 10.1007/9783-662-44654-6_14. hal-01391305

\section{HAL Id: hal-01391305 \\ https://hal.inria.fr/hal-01391305}

Submitted on 3 Nov 2016

HAL is a multi-disciplinary open access archive for the deposit and dissemination of scientific research documents, whether they are published or not. The documents may come from teaching and research institutions in France or abroad, or from public or private research centers.
L'archive ouverte pluridisciplinaire HAL, est destinée au dépôt et à la diffusion de documents scientifiques de niveau recherche, publiés ou non, émanant des établissements d'enseignement et de recherche français ou étrangers, des laboratoires publics ou privés.

\section{(c)(1)}

Distributed under a Creative Commons Attribution| 4.0 International License 


\title{
Profound Degree: a conservative heuristic to repair dynamic CSPs
}

\author{
Yosra Acodad • Amine Benamrane . \\ Imade Benelallam • El Houssine Bouyakhf
}

the date of receipt and acceptance should be inserted later

\begin{abstract}
For a better treatment of Dynamic Constraint Satisfaction Problems (DCSPs), several techniques have been developed to be used in repair algorithms. We cite, for example, the variables/values ordering heuristics and local search techniques.

We distinguish between static heuristics, which calculate their values once at the beginning of the search, and dynamic heuristics that use an expensive intelligence in terms of solving time.

In this paper, we propose a new static variable ordering heuristic, Profound Degree ( $p d e g)$, based on deg heuristic, which calculates the degree of influence of a given variable, on the whole constraints network, relatively to its position in the network.

We evaluate this heuristic on the Extended Partial-order Dynamic Backtracking $(E P B D)$ approach, which is an approach to repair DCSPs solutions, and we compare it to the best-known variables ordering heuristics $(\mathrm{VOHs})$ for repairing. The evaluation of performance is on random binary problems and meeting scheduling problems, with the criteria of computation time, number of constraints checks and Hamming distance between the former and the current solution.
\end{abstract}

\section{Introduction}

In recent years, several improvements have been made in algorithms for solving and repairing DCSPs [3] and [4]. Among these, we quote $V O H s$ [9], filtering techniques and conflicts analysis.

In fact, the order in which variables are affected by a search algorithm is crucial. Indeed, the use of different $V O H s$ to solve the same $C S P$ can lead to very different results in terms of performance.

Y. Acodad · A. Benamrane · E. H. Bouyakhf

LIMIARF. Faculty of Sciences Mohammed the fifth University - Agdal Rabat, Morocco

E-mail: yosra.acodad@gmail.com, benamraneamine@gmail.com, bouyakhf@fsr.ac.ma

I. Benelallam

INSEA, National Institut of Statistics and Applied Economic - Irfane Rabat, Morocco

E-mail: imade.benelallam@ieee.org 
The used heuristics can be classified into two broad categories: Static variables ordering heuristics $(\mathrm{SVOHs})$ and dynamic variables ordering heuristics (DVOHs). In this paper, we present a new $S V H O$, profound degree heuristic (pdeg), which is inspired by the deg heuristic insofar as it uses the concept of neighborhood. In effect, pdeg is not limited to calculate the number of neighbor connections of concerned variable, but it also measures the impact of its eventual disturbances (since we study this heuristic under correction of DCSPs) in relation to all constraints network, i.e., it takes into consideration all levels of the network neighborhoods, by giving each level a different weight, then it keeps intact the value of the variable having a strong impact, in order to reduce disturbing the network.

\section{Background}

2.1 Minimal Perturbation Problem $(M P P)$

Definition 1 (MPP) A Minimal Perturbation Problem $(M P P)$ is a triple $\Pi=$ $(\Theta, \alpha, \delta)$, where:

- $\Theta$ is a $C S P$.

- $\alpha$ is a partial or complete assignment for $\Pi$ that is called initial assignment.

- $\delta$ is a function that defines a distance between any two assignments.

A solution to a $M P P$ is a solution to $\Pi$ with minimal distance from $\alpha$ according to $\delta$.

Definition 2 (Distance set $D$ ) Let $\sigma$ and $\gamma$ be partial assignments for $\Theta$. V is the set of variables and $W(\sigma, \gamma)$ is the set of variables such that the value assignment for $v$ in $\sigma$ is different from the value assignment for $v$ in $\gamma$ :

$$
W(\sigma, \gamma)=\left\{v \in V \mid\langle v, v a l\rangle \in \sigma,\left\langle v, v a l^{\prime}\right\rangle \in \gamma, v a l \neq v a l^{\prime}\right\}
$$

$W(\sigma, \gamma)$ is called a distance set for $\sigma$ and $\gamma$ and the elements of the set are called perturbations.

Definition 3 (Function $\delta$ ) In an $M P P$, the distance function of some assignment $\sigma$ from $\alpha$ is defined as the cardinal of the set $D(\sigma, \alpha)$ :

$$
\delta(\sigma, \alpha)=|D(\sigma, \alpha)|
$$

Otherwise, $\delta$ is the hamming distance between $\sigma$ and $\alpha$.

Definition 4 (Solution Value Assignment $(S V A)) \alpha$ is the initial assignment of a $C S P$. For each variable $v_{i}$ :

$$
\text { If }\left\langle v_{i}, \operatorname{val}_{i_{j}}\right\rangle \in \alpha \Longrightarrow v_{i} . S V A=\operatorname{val}_{i_{j}}
$$

$S V A$ is the acronym of Solution Value Assignment. 
2.2 Extended Partial-order Dynamic Backtracking (EPDB)

Unlike several $C S P$ resolution approaches that use a fixed order of variables, the Partial-order Dynamic Backtracking $(P D B)[2]$ uses a partial order, that is built from nogoods and safety conditions, that is dynamically modified during the search process.

Definition 5 (nogood) A nogood is a failure justification. It's an expression of the form :

$$
\left(x_{1}=v_{1}\right) \wedge \ldots \wedge\left(x_{k}=v_{k}\right) \rightarrow x \neq v
$$

Definition 6 (safety conditions) A safety condition is defined as an assertion of the form: $x<y$, with $x$ and $y$ variables.

If $S$ is a set of safety conditions, we denote by $\leq_{s}$ the transitive closure of $<$, meaning that $S$ is acyclic if $\leq_{s}$ is antisymmetric.

$$
x<_{s} y \Longrightarrow x \leq_{s} y, y \not \leq_{s} x
$$

In another way: $x<_{s} y$, if there is a sequence (possibly empty) of safety conditions:

$$
x<z_{1}<z_{2}<\ldots<z_{n}<y
$$

When having to generate a nogood during the search, due to conflict situation (creating a nogood caused by a violated constraint) or domain wipe out (nogoods resolution), and when no order is imposed in existing nogoods and safety conditions between variables concerned with this conflict, there may be significant choices for the variable that will emerge as the generated nogood conclusion. The performance of this approach depends mainly on the quality of this choice, which is not covered in $P D B$.

The major goal of $E P D B$ is to extend the classic description of $P D B$, by exploiting its flexibility to repair assignments using repair-directed heuristics, when having to build an order between some variables, to control changes.

\section{Profound Degree Heuristic (pdeg)}

\subsection{Description of heuristic}

The deg $V O H$ [5] has a major advantage, it's that it does not compute every time dynamic information about the current state of research (such as the current domains size $d o m$ or the current variables degree $d d e g[10]$ ), but it operates static information.

However, in the context of repairing solutions, this heuristic appears limited. In fact, no information regarding the position of variables in relation to the entire network is operated, whereby the change of a variable can affect not only the neighbors, but all of the variables in the network with a given probability. For this, we propose to calculate an estimation of this information for each variable $X$ at the beginning of the research, considering all the network constraints, and by associating to each constraint a weight, higher or lower, in relation to its distance from $X$. pdeg is considered as the sum of those weights. 


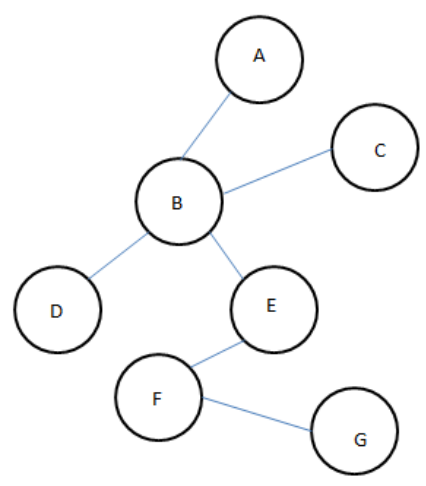

Fig. 1: A simple constraints network

Now consider the simple network above (figure 1). Suppose that each variable has a consistent value and due to a perturbation of the $D C S P$, the value of $E$ must be changed. Assume that all constraints have the same tightness $p_{2}$. Changing $E$ will generate the following probabilities $P$ :

$$
\begin{gathered}
P\left(\text { violate }\left(C_{E B}\right)\right)=P\left(\text { violate }\left(C_{E F}\right)\right)=p_{2} \\
P\left(\text { violate }\left(C_{B A}\right)\right)=P\left(\text { violate }\left(C_{B C}\right)\right)=P\left(\text { violate }\left(C_{B D}\right)\right)=P\left(\text { violate }\left(C_{F G}\right)\right)= \\
p_{2} * p_{2}=p_{2}{ }^{2}
\end{gathered}
$$

If the probabilities of violating constraints are considered as weights, knowing that $p_{2} \in[0,1]$, the weights of constraints network are:

$$
\begin{aligned}
& W \text { eight }\left(C_{E B}\right)_{/ E}=W \text { eight }\left(C_{E F}\right)_{/ E}=p_{2} \geqslant W \text { eight }\left(C_{B A}\right)_{/ E}= \\
& W \text { eight }\left(C_{B C}\right)_{/ E}=W \text { eight }\left(C_{B D}\right) / E=W \text { eight }\left(C_{F G}\right)_{/ E}=p_{2}{ }^{2}
\end{aligned}
$$

This reflects that, whenever the constraint $C$ is closer to the variable $X, C$ is more influenced by the change of $X$, since the variables concerned by $C$ are more influenced by changing $X$.

In fact, given two constraints $C_{1}$ and $C_{2}$, if the constraint $C_{1}$ is closer to $X$ than $C_{2}$, then $C_{1}$ has a cost in calculation of the heuristic value of $X$ which is more interesting than $C_{2}$, since changing $X$ engenders a risk of violating $C_{1}$ more important than violating $C_{2}$.

In reality, constraints networks are not as simple as that, they are quite as figure 2:

On the other hand, the hardness of constraints network (especially when the constraints used in the modeling are intent) is not always known, reasons why choosing to replace the weight, that corresponds to the tightness of the constraints, by an average hardness of $1 / 2$.

Inspired by this idea, $p d e g$ heuristic accumulates, for each variable $X$, the weights of all constraints. These weights are higher each time the constraints are closer to $X$. 


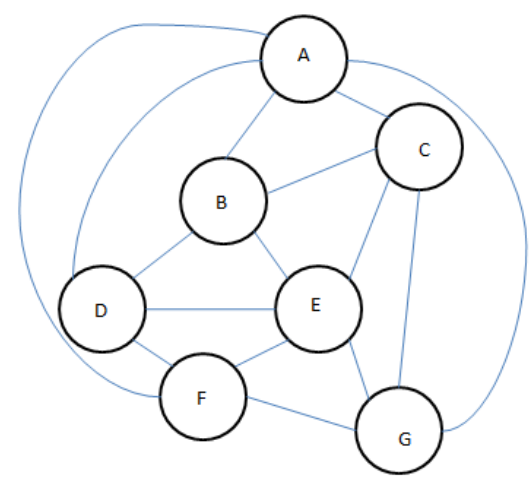

Fig. 2: A constraints network

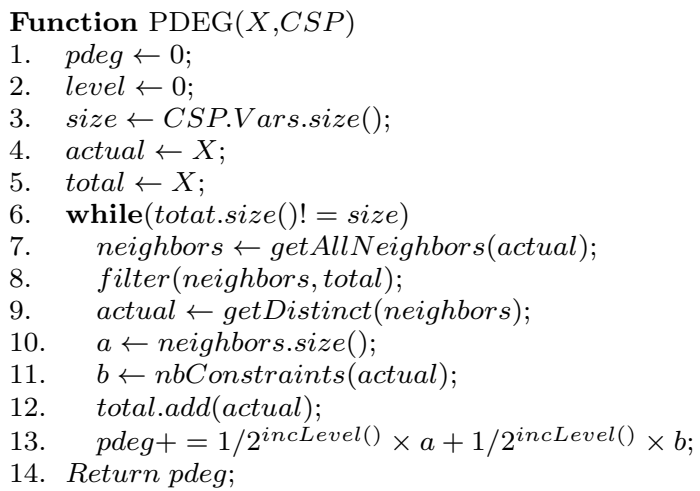

Fig. 3: Description of pdeg heuristic

\subsection{Execution of heuristic}

Both of the value of $p d e g$ and the level, that is incremented each time the constraint is far from the variable in question, are initialized by 0 (lines 1 and 2 ). The variable size contains the number of variables in the $C S P$ (line 3 ) and actual and total lists, initiated by the variable for which having to calculate the $p d e g$, serve respectively to store all variables in the current level and all variables already involved in the calculation (lines 4 and 5).

Will not accounting all constraints network variables, the heuristic calculates the neighborhood of the current variables, then this neighborhood is filtered to include only new variables, to not record a same constraint twice (lines 6-8).

Note that getAllNeighbors() (line 7) recognizes a neighbor many times as the number of constraints that bind it with the current variables.

The heuristic puts into actual current neighbors eliminating redundancies, in $a$ the number of neighbors of actual variables with redundancy (which is equivalent to the number of constraints with neighbors) not yet recognized and in $b$ the number of constraints between these neighbors (lines 9 and 11). At this point, it adds to total the current neighbors and to pdeg the weight of neighbors, which is $1 / 2$ 
to the power the level incremented, multiplied by the number of redundant neighbors, and it does the same thing for the number of constraints between neighbors, after incrementing the level (lines 12 and 13).

The heuristic leaves the while loop once all variables are accounted, which means it counted all the constraints of the network, and the value of pdeg is returned as the profound degree of the variable $X$ (line 14).

\subsection{Example of simulation}

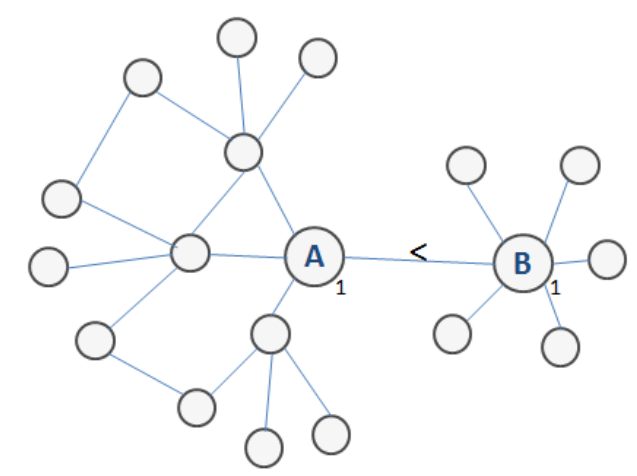

Fig. 4: Graph of constraints

We consider the constraints network above and assume that all variables have a consistent value $(A=1, B=1$ and all other variables have values consistent with the whole constraints network). We suppose that the DCSP has undergone a disturbance that is the change of the nature of the constraint $C_{A B}$, that was: $A=B$, and that became: $A<B$. To satisfy this constraint, the $E P D B$ algorithm $[1]$ is restarted in order to repair solution. $E P D B$ chooses to change the value of $A$, or the one of $B$, depending on the used heuristic.

Using $E P D B_{\text {deg }}$, heuristic values are:

$\operatorname{deg}(A)=4$

$\operatorname{deg}(B)=6$.

So the approach generates the nogood: $B=1 \Longrightarrow A \neq 1$, since it retains the value of the most relevant variable in the network, i.e., the one whose change can disrupt more the DCSP and delay resolution. For this reason, the value of $A$ is changed.

Now, using $E P D B_{\text {pdeg }}$, the values of used heuristic are:

$\operatorname{pdeg}(A)=1 / 2 \times 4+1 / 2^{2} \times 1+1 / 2^{3} \times 14+1 / 2^{4} \times 2=4,125$

$\operatorname{pdeg}(B)=1 / 2 \times 6+1 / 2^{2} \times 0+1 / 2^{3} \times 3+1 / 2^{4} \times 1+1 / 2^{5} \times 9+1 / 2^{6} \times 2=3,75$

Then the approach generates the nogood: $A=1 \Longrightarrow B \neq 1$ and changes the value of $B$. 


\section{Experimental results}

We carried out a series of experimental tests to compare the integration of pdeg heuristic, within the EPDB algorithm, to other $V O H s$.

Heuristics used for repairing are those that improve the best the behavior of $E P D B$, namely the degree $(\mathrm{deg})$, the domain size (dom), the number of conflicts (conf) and the ratio between the domain size and the weighted degree $(\mathrm{dom} / w \mathrm{deg})$ $[6]$.

We evaluate the performance in terms of constraints checking $(C C s)$, computing time (Time $(s c))$ and Hamming distance $(H D)[8]$ and [7].

All experiments were performed on the Java platform.

\subsection{Experiments on Randoms}

Random CSPs are characterized by a quadruplet $<n, d, p_{1}, p_{2}>$, where $n$ is the number of variables, $d$ the number of values per variable, $p_{1}$ the network connectivity (density) and $p_{2}$ the constraints tightness.

The density $p_{1}$ of a constraints graph is defined as the the ratio of the number of constraints relative to the maximum number of possible constraints in this network.

The tightness $p_{2}$ of a constraint corresponds either to the proportion of unauthorized tuples, or the probability that a tuple is not allowed.

Tests have been performed on sparse and dense problems, respectively < $20,10,0.25,0.6\rangle$ and $\langle 20,10,0.75,0.27>$. The constraints tightness is selected such as areas are as complex as possible while problems have solution.

For logical reasons, we assume that the maximum rate of added constraints is equivalent to $\sim 25 \%$. For each pair, 20 instances were solved using each heuristic, and the results are presented as an average of these 20 instances.

Figure 5 presents the effectiveness of heuristics applied to the $E P B D$, for the most complex regions of the low density selected. In terms of number of constraints tested CCs, Between $10 \%$ and $24 \%$ of new injected constraints, pdeg is the best, elsewhere, dom/wdeg and dom are the most efficient. Otherwise, the deg is the closest to pdeg, which seems the most stable.

In time graph, dom/wdeg recedes (also for dom but not in the same manner), due to the calculation of dom and wdeg heuristics, especially for wged. pdeg is almost always the best.

Concerning the Hamming distance $H D$, there is a competition between heuristics, and pdeg remains a good competitor in most regions.

Figure 6 shows the effectiveness of the heuristics for the most complex areas of the high density $p_{1}=0.75$. In terms of number of $C C s$ and execution time, pdeg usually looks the best although not improving too much the behavior of deg. Concerning Hamming distance, no heuristic is absolutely the best.

4.2 Experiments on Dynamic Meeting scheduling problems

A Meeting Scheduling Problem $M S P$ is characterized by $<m, p, n, d, h, t, a>$, where $m$ is the number of meetings, $p$ the number of participants, $n$ the number 

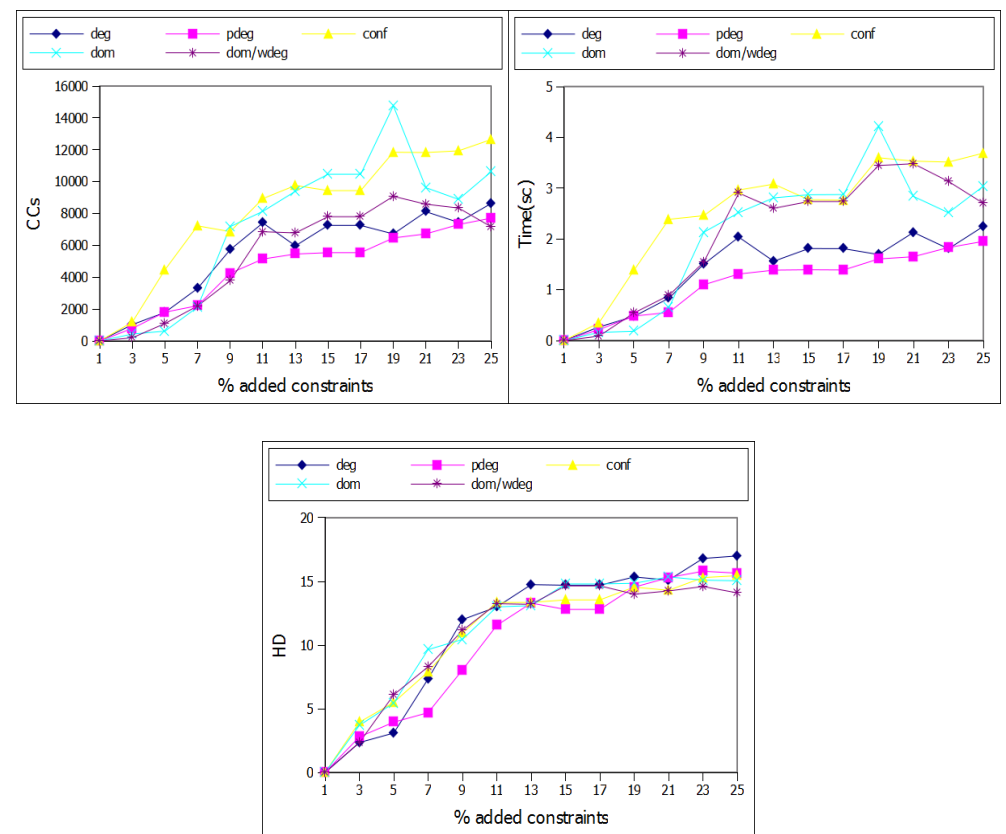

Fig. 5: Number of CCs, Execution time performed and HD $\left(p_{1}=0.25, p_{2}=0.60\right)$
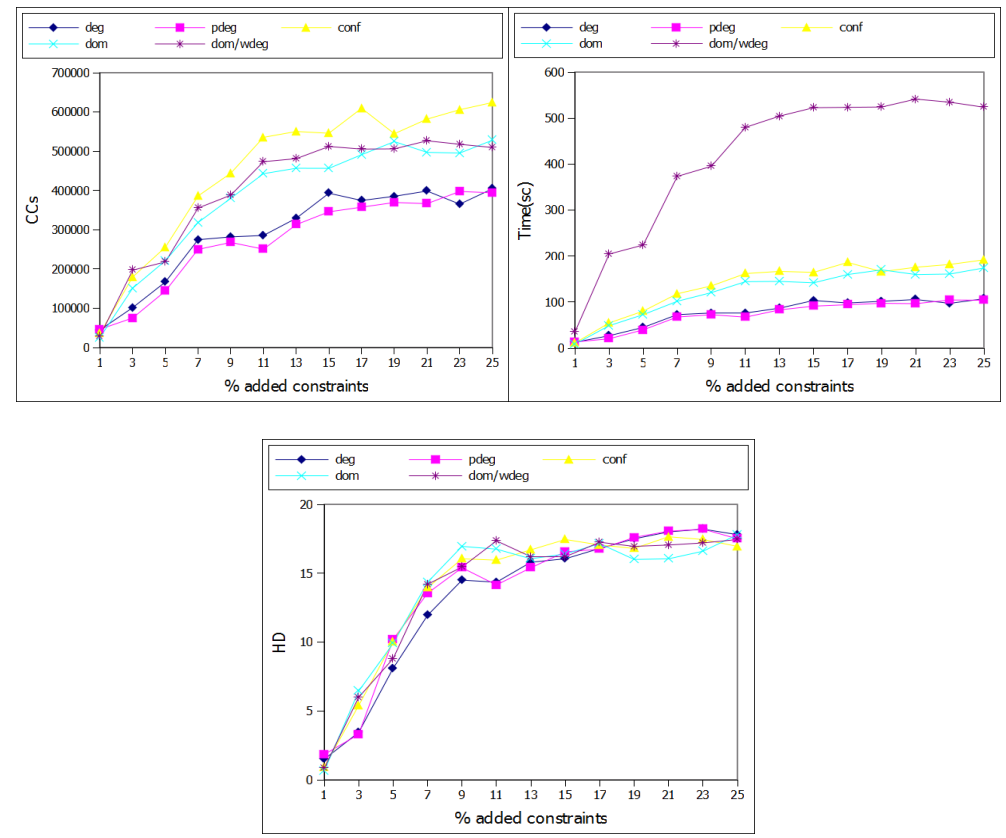

Fig. 6: Number of CCs, Execution time performed and HD $\left(p_{1}=0.75, p_{2}=0.27\right)$

of meetings per participant and $d$ the number of days. Different time slots are 
available for each meeting, and $h$ is the number of hours per day, $t$ the duration of the meeting and $a$ the percentage of availability for each participant.

We present our results for the class $<20,5,15,5,10,1,70>$ and we vary the rate of changed constraints from $\sim 1 \%$ to $\sim 25 \%$. We generated 20 different instances solved using each heuristic in $E P D B$.
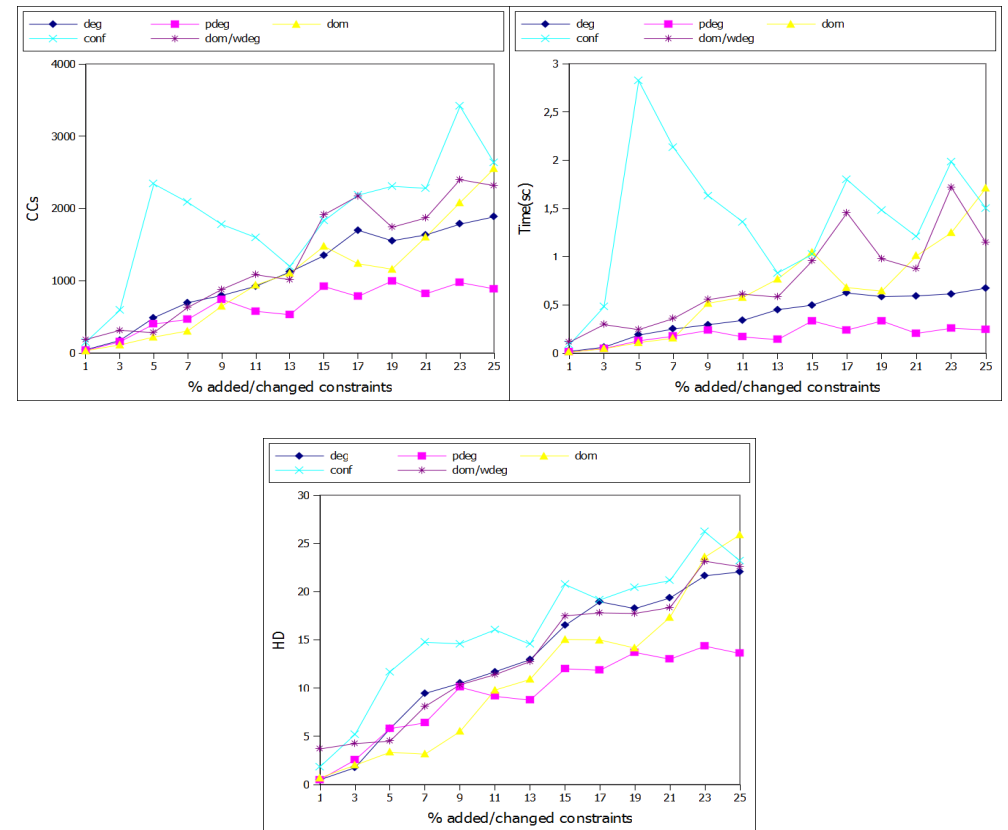

Fig. 7: Number of CCs, Execution time performed and HD

Figure 7 shows the effectiveness of the heuristics in terms of number of $C C s$, execution time and HD. pdeg leads to a great improvement in most areas (from $10 \%$ of disrupted constraints in terms of CCs, $7 \%$ in terms of time and $11 \%$ in terms of $H D)$.

\subsection{Discussion}

For low densities, pdeg behaves better than deg, more remarkably than high densities. In fact, in high densities, almost all variables are connected, i.e., for each given variable, almost all other variables in the network are its neighbors. Therefore calculating pdeg of a variable almost returns to calculate its deg.

$p d e g$ leads, especially for low densities, to a minimum Hamming distance $(H D)$. In fact, the idea of pdeg is to change the value of the least constraining variable in the network, i.e., the one that, when changing its value, will cause the least change to other variables. And knowing that the variables before the correction have $S V A s$ values, so a minimum change of network variables is a minimum change of $S V A s$, then a maximum of $S V A s$. 
Although the heuristics dom, conf and dom/wdeg, in some regions, do less constraints checking than static heuristics deg and pdeg, they consume more computational time because of their dynamic aspect.

\section{Conclusion}

In this paper, we introduce a new $V O H, p d e g$ heuristic, for estimating the influence of variables in the whole constraint network, in the process of repairing solutions in dynamic environments.

Based on the results of experiments on a wide class of problems, we found and proved that this heuristic, used in DCSPs correction, exceeds the heuristics deemed to be efficient when solving CSPs.

In fact, the idea of pdeg heuristic is to disturb only the parts of the constraints network, whose probability of influence is minimal. Indeed, for low to medium densities, as seen in the random problems with density $p_{1}=0,25$ and the $M S P$ (and especially for medium densities), using pdeg improves remarkably the repair of solution, not only in relation to research performance (time and $C C s$ ), but also with respect to the quality of the solution (Hamming distance).

As perspectives, we intend to integrate this heuristic in other algorithms for solving and repairing centralized and distributed $C S P s$, to hybridize it with other heuristics according to areas in which they show a remarkable improvement in order to lead to an optimal heuristic, and to experiment it on real problems.

\section{References}

[1] Y. Acodad, I. Benelallam, S. Hammoujan, and E. H. Bouyakhf. Extended Partial-order Dynamic Backtracking algorithm for dynamically changed environments. (ICTAI), 2012 IEEE 24th International Conference on 7 Nov. 2012: 580-587.

[2] M. L. Ginsberg and D. A. McAllester, Gsat and dynamic backtracking. Journal of Artificial Intelligence Research, vol. 1, pp. 25-46, 1994

[3] R. Dechter and A. Dechter, Belief maintenance in dynamic constraint networks. in AAAI, 1988, pp. 37-42.

[4] C. Bessiere. Arc-consistency in dynamic constraint satisfaction problems. In Proc. AAAI'91, pages 221-226. AAAI Press, 1991.

[5] R. Dechter and I. Meiri. Experimental evaluation of preprocessing techniques in constraint satisfaction problems. In Proceedings of IJCAI'89, pages 271-277, 1989.

[6] F. Hemery, C. Lecoutre and L. Sais. 2004. Boosting systematic search by weighting constraints. ECAI. August.

[7] R. Zivan, G. Alon and M. Amnon. 2011. Hybrid search for minimal perturbation in Dynamic CSPs. Constraints 16, no. 3: 228-249.

[8] E. Hebrard, O. Barry and T. Walsh. 2007. Distance Constraints in Constraint Satisfaction. IJCAI. January 6.

[9] J. Ortiz-Bayliss, H.Terashima-Marin, O. Ender, J. Andrew and E. Santiago. 2013. Exploring heuristic interactions in constraint satisfaction problems: A closer look at the hyperheuristic space. Evolutionary Computation (CEC), 2013 IEEE Congress on. IEEE, June 20.

[10] C. Bessiere and R. Jean-Charles. 1996. MAC and combined heuristics: Two reasons to forsake FC (and CBJ?) on hard problems. Principles and Practice of Constraint Programming CP96. Springer Berlin Heidelberg, January 1. 